\title{
Transatlantica
}

Revue d'études américaines. American Studies Journal

\section{Fiction historique : enjeux théoriques et idéologiques}

Université de Paris (Paris-Diderot), 21 juin 2019

\section{Patrice Dallaire}

\section{(2) OpenEdition \\ Journals}

\section{Édition électronique}

URL : https://journals.openedition.org/transatlantica/15127

DOI : $10.4000 /$ transatlantica. 15127

ISSN : $1765-2766$

\section{Éditeur}

Association française d'Etudes Américaines (AFEA)

\section{Référence électronique}

Patrice Dallaire, «Fiction historique : enjeux théoriques et idéologiques », Transatlantica [En ligne], 2 | 2019, mis en ligne le 01 octobre 2020, consulté le 31 janvier 2023. URL : http://

journals.openedition.org/transatlantica/15127 ; DOI : https://doi.org/10.4000/transatlantica.15127

Ce document a été généré automatiquement le 31 janvier 2023.

\section{(c) (i) (9)}

Creative Commons - Attribution - Pas d'Utilisation Commerciale - Pas de Modification 4.0 International - CC BY-NC-ND 4.0

https://creativecommons.org/licenses/by-nc-nd/4.0/ 


\title{
Fiction historique : enjeux théoriques et idéologiques
}

\author{
Université de Paris (Paris-Diderot), 21 juin 2019
}

\section{Patrice Dallaire}

1 Dans le cadre du projet «Faire le point sur les fictions historiques» (https:// mhma.hypotheses.org/133) était présentée, le 21 juin dernier, la journée d'études intitulée «Fiction historique: enjeux théoriques et idéologiques / Theoretical and Ideological Issues in Historical Fiction", à l'Université de Paris, sur son campus Paris Rive Gauche. Réunissant une trentaine de chercheurs, les quatre sessions ont permis d'entendre huit présentations couvrant des champs aussi divers que la littérature, le théâtre, le cinéma, les séries télévisées ou encore la danse.

Organisée sous l'égide du LARCA (Laboratoire de recherches sur les cultures anglophones) UMR 8225, avec le soutien de l'université de Liverpool, la journée avait pour objectif d'aborder la thématique de la fiction historique par le biais de regards pluridisciplinaires, comme le soulignent les organisatrices, les professeures Laurence Cros (Université de Paris) et Marie-Jeanne Rossignol (Université de Paris). Elles invitent les historiens à surmonter leur réticence, presque instinctive, vis-à-vis de la fiction et à aborder les produits culturels comme objets légitimes de recherches historiques. Certes, elles reconnaissent que les raccourcis historiques, qui marquent certaines œuvres de fiction, ont parfois pour effet de court-circuiter la recherche de la vérité historique, en lui substituant une scénarisation dramatique dont le plus fâcheux défaut est sans doute de s'immiscer dans la mémoire collective d'un vaste public pour lequel il " constitue la principale, voire l'unique, porte d'entrée à la connaissance du passé ».

En se penchant sur la comédie musicale Hamilton (dont le vif succès populaire à New York a été suivi d'une tournée américaine puis, à partir de 2020, d'une tournée mondiale), la première session a d'entrée de jeu lancé le débat en illustrant à la fois les avantages et les inconvénients de la fiction historique. Cheryl Hudson (University of Liverpool), dans son propos "Who lives, who dies, who tells your story? Alexander Hamilton as culture warrior ", soutient qu'il importe finalement peu de savoir qui écrit le récit historique ou même quelle histoire y est racontée. Ce qui importe plutôt, c'est 
que cette comédie musicale «nous renseigne sur la nature de l'Histoire elle-même, sur les rapports entre art et histoire et entre vérité et fiction, ainsi que sur la façon dont cette relation se réécrit sous nos yeux » dans un espace public de plus en plus marqué par des «guerres culturelles » (culture wars). Selon Hudson, la relation entre la vérité et la fiction n'est pas compliquée, quoique complexe et empreinte de nuances. La fiction historique permet en effet de faire rejaillir des vérités d'ordre symbolique ainsi que des valeurs universelles, au travers d'une compréhension de l'histoire qui se transforme avec le temps et notre vision changeante du passé.

4 L'accueil de la comédie musicale Hamilton par les historiens a été mitigé, voire hostile. Certains, de manière militante, croient devoir débusquer toutes les erreurs qu'on peut y trouver et corriger le tir avant que celles-ci ne prennent valeur de vérité historique. Pour Hudson, ils font fausse route : lapidaire, elle affirme que les « historiens feraient mieux de redoubler d'efforts [et] de balayer devant leur porte avant de se plaindre d'une comédie musicale à succès ».

5 Au cours des dernières années aux États-Unis, l'histoire a été instrumentalisée (elle utilise le mot weaponized) dans un contexte de divisions sociales et politiques marqué par les "guerres culturelles" entre conservateurs et libéraux: ces derniers se disputent l'héritage politique de la Constitution et les valeurs qui lui sont attachées. Celles-ci ne seraient plus ni universelles, ni partagées, minant ainsi l'identité américaine traditionnelle. Mais quel est le rôle des historiens dans ces débats? Doivent-ils s'interposer comme médiateurs des "guerres culturelles»? Doivent-ils accepter l'idée que la vérité n'est pas seulement empirique mais nourrie, aussi, de mythes dictés par l'imagination des uns et des autres? Pour Hudson, si l'on accepte l'idée de l'auteure de romans historiques Hilary Mantel pour qui le mythe est non un mensonge mais une vérité enrobée de symboles et de métaphores, l'historien peut alors adopter une perspective plus détachée lui permettant de reconnaitre des «qualités transformatives " à l'histoire américaine. Ainsi, selon Hudson, chaque génération a réinterprété la Révolution de 1776 en menant des combats s'en réclamant, de l'abolitionisme à la guerre de Sécession, puis à la lutte pour les droits des femmes et plus récemment pour les droits et libertés civiques - et ce jusqu'à la fin du "grand récit national » qu'elle situe en 1989. En ce sens, Hamilton ouvre une nouvelle porte sur l'étude du passé et provoque de nouvelles interrogations sur le sens que l'on doit donner à l'expérience politique américaine. Elle conclut en notant que, cependant, la question fondamentale demeure: est-il possible de concilier les droits des minorités avec la volonté et les demandes de la majorité démocratique ? En dressant une esquisse de la place qu'occupe Hamilton dans les débats actuels sur la relation qu'entretiennent les Américains avec leurs mythes fondateurs et les transformations du grand récit national américain, Hudson réussit son pari d'interroger la nature même de l'histoire et sa relation à l'entreprise artistique. Ici, à notre avis, on ne fait plus vraiment la différence entre l'homme et la comédie musicale éponyme, et c'est sans doute ce qui était recherché par l'auteur de la pièce, Lin-Manuel Miranda.

6 La communication suivante, par Caitlin O'Keefe (Sarah Lawrence College), «Hamilton's Angelica Schuyler and the Question of Ahistorical Feminism in Historical Fiction ", faisait naturellement suite à celle de Hudson et en approfondissait certains des enjeux. O'Keefe nous montre comment Luis-Manuel Miranda, le créateur de la comédie musicale, invente un scénario où Angelica Schuyler, sœur d'Eliza, l'épouse de Hamilton, sacrifie sa communion intellectuelle et son attirance mutuelle avec ce 
dernier : œuvrant au bonheur de sa sœur par sens du devoir familial, Angelica veut trouver à Eliza un mari et, ce faisant, se «brise elle-même le cœur ». En offrant une place centrale à ces personnages - femmes, esclaves ou minorités - habituellement relégués aux marges du récit historique, la comédie musicale Hamilton non seulement met en scène un féminisme anhistorique mais offre une image romancée, voire carrément fabriquée, de Hamilton et de sa relation avec Angelica Schuyler. Il est clair, en écoutant Caitlin O'Keefe, que Miranda, comme scénariste, en épousant l'impératif dramaturgique de la romance et en construisant des personnages parfois détachés de la réalité historique, a délibérément opté pour une histoire susceptible de rencontrer l'adhésion du public d'aujourd'hui. Ainsi, nous dirions que Miranda s'est plié au vieil adage: "Why let facts stand in the way of a good story? " Mais comme O'Keefe le rappelle, les lacunes historiques et théoriques de Hamilton n'empêchent pas de reconnaître ses mérites en tant que démarche artistique et historique.

7 Avec «Dancing History: Fiction and Experiment", Jerome de Groot (University of Manchester) nous entraîne sur une autre scène, au sens propre comme figuré. Citant le critique littéraire Fredric Jameson, il considère que les fictions historiques jouent un rôle dans la définition du "réalisme » et qu'elles sont intimement liées à la pratique historique. Ainsi, le roman historique, dans la tradition occidentale de ce réalisme, répond à des critères d'authenticité et de vraisemblance (believability). Selon De Groot, ce genre littéraire interpelle particulièrement le lecteur car il requiert de lourds efforts d'imagination, en particulier pour se transporter dans le temps. Dans son analyse de la relation entre l'histoire, l'identité et le corps, De Groot prend le roman Swing Time de Zadie Smith pour témoin de l'innovation musicale afro-américaine que constitue le jazz. Ce roman, ainsi que le film éponyme de 1936 qui l'a inspiré, réinsèrent similairement le corps africain dans l'histoire. La danse, à la fois chargée de signification et « un peu ridicule », est aussi multidimensionnelle, multi-temporelle, le corps et le mouvement au présent permettant de se transporter dans les siècles et dans l'espace. La danse établit des liaisons avec le passé et les lieux qui habitent notre mémoire; elle est aussi méditation sur le patrimoine identitaire et l'histoire, sans que l'on puisse pour autant, précise-t-il, établir une linéarité.

8 De Groot en conclut que la danse ne constitue que l'une des façons pour le romancier historique d'expérimenter avec la subjectivité, le corps, le temps et la notion de race. En tant que genre aujourd'hui de premier-plan, « l'expérimentation » nous oblige donc à « suivre la cadence pour mieux saisir le but recherché ».

9 Dans une communication intitulée « La fiction comme avant-garde du renouvellement historiographique et de sa diffusion dans la culture populaire? Les réécritures féministes des épopées antiques depuis les années 1990 », Maureen Attali (Université Rennes 2) présente un genre de fiction, écrit par des femmes, porté par une perspective féministe et centré sur des personnages féminins jusqu'ici secondaires. Relevant d'un projet académique autant que littéraire, cette perspective est d'après elle entrée en pleine effervescence depuis les années 1980. Attali s'est penchée sur trois œuvres de ce type, publiées au cours de la dernière décennie, soit: Lavinia, Circe, The Silence of the Girls, des autrices américaines Ursula K. Le Guin et Madeline Miller et de la britannique Pat Barker. Elle y ajoute également un corpus iconographique, trop souvent négligé par les historiens. Non seulement Maureen Attali marque son désaccord avec les critiques de la réécriture d'épopées, lesquels s'insurgent contre les modifications apportées à des classiques, mais elle démontre également que ces réécritures satisfont pleinement aux 
canons du genre. Pour elle, la valeur de ces fictions provient de leurs processus de réécriture, à partir d'une perspective féministe, qui offrent une toute nouvelle version de l'histoire. Cependant la réécriture seule ne suffit pas nécessairement à la transposition d'une expérience féministe; encore faut-il que le thème s'y prête, telles la violence genrée ou l'objectification des femmes, souvent ramenées à leur apparence physique. Plus intéressantes encore sont, selon elle, les notions de mariage arrangé et de viol (présentes dans les trois œuvres), ou la liminalité des personnages féminins et leur rôle comme intermédiaires entre les dieux et les mortels. Bien sûr, Attali reconnaît d'emblée aux trois œuvres des objectifs pédagogiques et idéologiques. Elles viennent remédier au déséquilibre entre les genres dans des récits millénaires, contrer les clichés et les idées préconçues sur la place des femmes dans les civilisations antiques et, surtout, lutter contre l'appropriation de ces textes par des relais conservateurs cherchant à légitimer un rôle secondaire censément dévolu aux femmes au cours des âges et toujours applicable dans nos sociétés contemporaines. Et si à la fin la dichotomie entre l'épopée et la vérité historique n'est jamais résolue, Attali nous enseigne que les réécritures ont le mérite de remettre "en cause l'existence d'une vérité historique objective, [tout] comme le font les historiens [traditionnels] actuels ».

Dans «Une fiction historique et sa réponse comme opposition idéologique : de 300 de Frank Miller à Three de Kierron Gillen", Benjamin Norguet (Université Paris 8) propose une étude comparée de deux œuvres, dont la seconde a été expressément rédigée en réponse à la première. Miller, que Norguet présente comme un polémiste aux positions tranchées, offre une version romantisée et fantastique, associant le lugubre (gloom) à la fantaisie - une œuvre dont l'adaptation cinématographique a donné lieu à encore plus d'exagérations, selon lui. Sa formation de philosophe amène Benjamin Norguet à s'interroger sur la notion même, en apparence oxymorique, de «fiction historique ». En utilisant certains personnages historiques, Kierren voulait pour sa part proposer une "histoire miroir", un anti-300, pour en déconstruire l'héroïsme et en inverser l'imagerie spartiate machiste. En opposant au «mythe fondateur » porté par 300 une anti-histoire, une fiction comme mémoire des opprimés, Kierren vise à renverser et à détourner les « codes des dominants » et à proposer une histoire du point de vue du peuple. Il fait ainsi revivre, par le biais de sa fiction, l'histoire des opprimés du monde - histoire disparue avec eux. Comme quoi, dirionsnous, l'adage voulant que l'histoire soit écrite par les vainqueurs trouve, pour une rare et rafraîchissante fois, son opposant « don quichottesque ».

11 Victor Faingnaert (Université de Caen), reconnait d'emblée que les fictions historiques offrent des possibilités intéressantes pour les historiens. Dans « Rome, une fiction entre recherches historiques et représentations culturelles ", sa position d'analyse se veut critique, cependant, des erreurs factuelles avérées, du moins celles que l'on pourrait relever, selon lui, à partir d'une analyse au prisme des cultural studies. Faingnaert rapporte que les consultants historiques de la série télévisée, coproduite par HBO et la $\mathrm{BBC}$, ont tous déploré avoir vécu des expériences pénibles avec les producteurs de la série. Apparemment, leur influence sur le scénario ou les décors s'est avérée minime, avec pour résultat une "Antiquité fantasmée » qui ne peut que déplaire aux historiens. Donc, puristes, s'abstenir! Sachant que la série reste relativement proche de sources comme La guerre des Gaules de Jules César, et malgré les raccourcis historiques souvent empruntés, le spectateur peut quand même, selon Faingnaert, dégager une idée assez 
fidèle des stratégies de César et comprendre l'évolution générale de la trame historique.

Il conclut son propos en soulignant que Rome était un projet ambitieux, trop peut-être, qui peut quand même être utile à l'historien: Rome, malgré ses carences et son caractère fictif, « est une véritable série historique [...] mais également une production historique, cherchant à représenter les possibles réactions et impacts des différents événements de la fin de la République romaine et des guerres civiles sur le quotidien du peuple », bref, une histoire par le bas qui rend la trame historique plus accessible, dans un sens. À notre avis, il est probablement inévitable que, dans un monde télévisuel où règnent la dictature des audiences et les impératifs de rentabilité commerciale, les scénaristes utilisent des recettes pour fidéliser le public, notamment en véhiculant des images historiques que connaît déjà le public et auxquelles il peut s'identifier ou, encore, en utilisant des codes culturels et des références permettant au spectateur de rapidement saisir les enjeux historiques.

13 Toujours dans le domaine des séries télévisées, Alban Gautier (Université de Caen), historien médiéviste, dans « Représentations du fait religieux dans les séries Vikings et The Last Kingdom », nous amène sur le terrain de l'adaptation télévisuelle d'une œuvre romanesque existante (The Last Kingdom) et d'une fiction pure et simple (Vikings). Les deux séries ont pour thème commun les contacts entre les peuples vikings et les peuples européens, en particulier via le prisme du religieux. Au-delà de ce constat de départ, Gautier se pose deux questions: ce qu'on voit dans ces deux séries est-il conforme à ce que l'on sait de la période ? Par-delà les erreurs et simplification, la fiction historique parvient-elle à saisir de manière originale et intelligente la réalité d'une époque ? En d'autres termes, arrive-t-elle à restituer ce qui est plausible et peutelle légitimement satisfaire les critères de l'authenticité ? Gautier est sans appel sur les nombreux anachronismes dont souffrent ces deux séries, tant en ce qui a trait aux sources qu'à la rencontre des deux univers culturels en présence, le paganisme et le christianisme. De même, la présentation des mythes nordiques, pourtant au cœur de la série, pèche aussi par anachronisme, parfois de plusieurs siècles. Gautier relève une volonté sous-jacente, dans toutes les saisons des deux séries qu'il a analysées, de mettre en avant non pas un simple biais anti-chrétien mais plutôt un "anticatholicisme intemporel hypocrite ", où se réfléchissent tous les préjugés de la Réforme à l'égard de l'Église romaine, de ses rites et de ses représentants, tous "érigés en repoussoir ". Inversement, le paganisme est constamment présenté comme empreint de sagesse primitive et le polythéisme comme particulièrement ouvert aux femmes. Alban Gautier est forcé de conclure que le discours véhiculé par la série est marqué par les tropismes concurrents du «boréalisme" et du "protestantisme ». Gautier ne nous dit pas, cependant, si ces représentations caricaturales du catholicisme médiéval traduisent inconsciemment de vieux préjugés des auteurs de la série ou si elles sont délibérées.

David Da Silva (Université Paris III Sorbonne-Nouvelle et Institut international de l'image et du son), qui s'intéresse à la culture populaire, ferme la dernière session en présentant une analyse intitulée «Le film historique hollywoodien à l'aune des cultural studies ». Au départ, il précise que la notion de cultural studies a évolué pour englober les gender, queer, native, African-American studies. Il est important de garder à l'esprit, selon Da Silva, que les œuvres cinématographiques doivent être analysées en tenant compte de la production et de l'intention du message véhiculé (son "encodage») et, en parallèle, de la réception de ce message par le public (son «décodage»). Dès lors, la 
réception d'une œuvre dépend beaucoup de ses publics. Da Silva a décortiqué deux films : Lincoln de Spielberg et Birth of a Nation de Nate Parker. De façon paradoxale, et malgré les intentions a priori nobles des réalisateurs, diverses minorités ont contesté vivement leur représentation dans ces productions. Au lieu d'atteindre les objectifs d'apaisement désirés, les films auraient plutôt attisé les conflits sociaux et attiré les critiques des femmes, des minorités visibles, ethniques, religieuses ou sexuelles. Selon Da Silva, les œuvres cinématographiques hollywoodiennes que l'on pourrait qualifier d'historiques avaient, jusqu'à présent, péché par leurs erreurs historiques et leur ethnocentrisme WASP. Il présente aussi des analyses sommaires de plusieurs autres productions hollywoodiennes traitant de personnages ou groupes opprimés, telles Imitation Game, Alexandre, Marie-Antoinette, Marie-Madeleine, Hidalgo ou encore Free State of Jones. Ces productions ont pris le contrepied de l'historiographie traditionnelle en proposant des visions alternatives du passé. Toutefois, elles ont souvent échoué à satisfaire les revendications politico-historiques des groupes concernés. Pour Da Silva, l'accueil négatif de ces films par les organisations militantes contraste avec celui du public, du moins tel que mesuré par le site internet IMDb. Ce qui amène Da Silva à un constat: les cultural studies, qui devaient proposer « une nouvelle histoire des ÉtatsUnis, débarrassée des préjugés du groupe dominant », ont failli à la tâche en imposant par rectitude politique "des films historiquement aussi faux que ceux du siècle précédent ", accentuant ainsi les conflits au lieu de les aplanir.

Si la rencontre a permis de présenter la fiction historique sous divers regards disciplinaires, on regrettera que les présentations n'aient pas plus systématiquement souligné les réels dangers de la manipulation de l'histoire - dangers qui accompagnent, parfois subtilement et involontairement mais, aussi, dans certains cas, avec un agenda politique plus ou moins avoué, certaines œuvres de fiction. Bref, c'est une question qu'il faudrait approfondir. Enfin, on peut légitimement se demander si les dommages à la vérité historique, dans la mémoire collective, par exemple, d'une comédie musicale à succès comme Hamilton, ne sont pas plus importants que les bénéfices résiduels émanant d'un regain d'intérêt, sinon pour l'Histoire, pour la recherche historique professionnelle. Espérons pouvoir en débattre dans les futures journées d'études du projet. Poser la question, souhaiter une réflexion sur ce choix, un peu cornélien pour les puristes, assez nouveau pour l'historien, de voir son métier rejoindre enfin le grand public, au prix, cependant, de souffrir que ses méthodes soient ignorées, dans une quête d'effets dramatiques qui travestit l'histoire elle-même ouvre un débat en soi. C'est tout l'avenir de la profession qui est en quelque sorte interrogé par la transformation des moyens de produire et de diffuser l'histoire.

Au moment où la société américaine vit à l'heure des « alternative facts ", quel rôle la fiction joue-t-elle dans la diffusion de versions biaisées de l'histoire et comment se prémunir contre leurs répercussions sociopolitiques insidieuses sur un public qui, souvent, ne demande qu'à être diverti ? Faudra-t-il que les historiens chevronnés se lèvent et décortiquent la fiction historique, sous toutes ses formes, pour en déceler les failles et protéger le public contre ses dangers cachés? 
INDEX

Thèmes : Actualité de la recherche

\section{AUTEUR}

PATRICE DALLAIRE

Université Laval et Université de Paris, LARCA, CNRS, F-75013 Paris 